\title{
Correction to: Propagation of Exponential Phase Space Singularities for Schrödinger Equations with Quadratic Hamiltonians
}

\section{Evanthia Carypis ${ }^{1} \cdot$ Patrik Wahlberg $^{2}$}

Published online: 8 April 2021

(c) Springer Science+Business Media, LLC, part of Springer Nature 2021

\section{Correction to: Journal of Fourier Analysis and Applications (2017) 23:530-571 \\ https://doi.org/10.1007/s00041-016-9478-6}

Our paper [1] contains an error in the proof of Proposition 8.1. More precisely the estimate claimed in Eq. (8.3) is erroneously motivated. In the following we state and prove Proposition 8.1 correctly.

We need the Hermite functions

$$
h_{\alpha}(x)=\pi^{-\frac{d}{4}}(-1)^{|\alpha|}\left(2^{|\alpha|} \alpha !\right)^{-\frac{1}{2}} e^{\frac{|x|^{2}}{2}} \partial^{\alpha} e^{-|x|^{2}}, \quad x \in \mathbf{R}^{d}, \quad \alpha \in \mathbf{N}^{d},
$$

and formal series expansions

$$
f=\sum_{\alpha \in \mathbf{N}^{d}} c_{\alpha} h_{\alpha}
$$

where $\left\{c_{\alpha}\right\}$ is a sequence of coefficients defined by $c_{\alpha}=c_{\alpha}(f)=\left(f, h_{\alpha}\right)$. The Hermite functions $\left\{h_{\alpha}\right\}_{\alpha \in \mathbf{N}^{d}} \subseteq L^{2}\left(\mathbf{R}^{d}\right)$ is an orthonormal basis.

Langenbruch [4, Theorem 3.4] has shown that the family of Hilbert sequence spaces

$$
\ell_{s, r}^{2}=\ell_{s, r}^{2}\left(\mathbf{N}^{d}\right)=\left\{\left\{c_{\alpha}\right\}:\left\|c_{\alpha}\right\|_{\ell_{s, r}^{2}}=\left(\sum_{\alpha \in \mathbf{N}^{d}}\left|c_{\alpha}\right|^{2} e^{2 r|\alpha| \frac{1}{2 s}}\right)^{\frac{1}{2}}<\infty\right\}
$$

The original article can be found online at https://doi.org/10.1007/s00041-016-9478-6.

$凶$ Patrik Wahlberg

patrik.wahlberg@lnu.se

Evanthia Carypis

ecarypis@gmail.com

1 Department of Mathematics, University of Turin, Via Carlo Alberto 10, 10123 Torino, TO, Italy

2 Department of Mathematics, Linnæus University, 35195 Växjö, Sweden 
for $r>0$ yields a family of seminorms for $\Sigma_{s}\left(\mathbf{R}^{d}\right)$ that is equivalent to the family (2.3) for all $h>0$, when $t=s>\frac{1}{2}$. Thus $\Sigma_{s}\left(\mathbf{R}^{d}\right)$ can be identified topologically as the projective limit

$$
\Sigma_{s}\left(\mathbf{R}^{d}\right)=\bigcap_{r>0}\left\{\sum_{\alpha \in \mathbf{N}^{d}} c_{\alpha} h_{\alpha}:\left\{c_{\alpha}\right\} \in \ell_{s, r}^{2}\right\} .
$$

Lemma If $s>\frac{1}{2}$ and $h>0$ then

$$
\sup _{x \in \mathbf{R}}\left|\partial^{k}\left(e^{-\frac{1}{2} x^{2}}\right)\right| \leqslant C_{h, s} h^{k} k !^{s}, \quad k \in \mathbf{N}
$$

where $C_{h, s}>0$.

Proof It is clear that

$$
\partial^{k}\left(e^{-\frac{1}{2} x^{2}}\right)=p_{k}(x) e^{-\frac{1}{2} x^{2}}
$$

where $p_{k}$ is a polynomial of order $k \in \mathbf{N}$. By induction one can prove the formula

$$
p_{k}(x)=k ! \sum_{m=0}^{\lfloor k / 2\rfloor} \frac{x^{k-2 m}(-1)^{k-m}}{m !(k-2 m) ! 2^{m}} .
$$

Since $k ! \leqslant 2^{k}(k-2 m) !(2 m)$ ! we can estimate

$$
\left|p_{k}(x)\right| \leqslant \sum_{m=0}^{\lfloor k / 2\rfloor} \frac{|x|^{k-2 m}(2 m) !}{m ! 2^{m-k}} \leqslant \sum_{m=0}^{\lfloor k / 2\rfloor}|x|^{k-2 m} m ! 2^{m+k}
$$

Combining with $m !=m !^{2 s-\varepsilon}$ where $\varepsilon=2 s-1>0$, this gives for any $h>0$ and $b>0$

$$
\begin{aligned}
\left|p_{k}(x)\right| h^{-k} k !^{-s} & \leqslant \sum_{m=0}^{\lfloor k / 2\rfloor}|x|^{k-2 m} m !^{2 s-\varepsilon} 2^{m+k} h^{-k} k !^{-s} \\
& =\sum_{m=0}^{\lfloor k / 2\rfloor}\left(\frac{\left(\frac{b}{s}|x|^{\frac{1}{s}}\right)^{k-2 m}}{(k-2 m) !}\right)^{s}\left(\frac{b}{s}\right)^{s(2 m-k)}\left(\frac{(k-2 m) ! m !^{2}}{k !}\right)^{s} \frac{2^{m+k} h^{-k}}{m ! \varepsilon} \\
& \leqslant e^{b|x|^{\frac{1}{s}}}\left(2\left(\frac{s}{b}\right)^{s} h^{-1}\right)^{k} \sum_{m=0}^{\lfloor k / 2\rfloor}\left(\frac{\left(2\left(\frac{b}{s}\right)^{2 s}\right)^{\frac{m}{\varepsilon}}}{m !}\right)^{\varepsilon}
\end{aligned}
$$




$$
\begin{aligned}
& \leqslant e^{\varepsilon\left(2\left(\frac{b}{s}\right)^{2 s}\right)^{\frac{1}{\varepsilon}}} e^{b|x|^{\frac{1}{s}}}\left(4\left(\frac{s}{b}\right)^{s} h^{-1}\right)^{k} \\
& =C_{h, s} e^{s 4^{\frac{1}{s}} h^{-\frac{1}{s}}|x|^{\frac{1}{s}}}
\end{aligned}
$$

where we pick $b=s 4^{\frac{1}{s}} h^{-\frac{1}{s}}$ in the last equality, and $C_{h, s}>0$. Thus since $\frac{1}{s}<2$

$$
\sup _{x \in \mathbf{R}}\left|\partial^{k}\left(e^{-\frac{1}{2} x^{2}}\right)\right| \lesssim h^{k} k !^{s} \sup _{x \in \mathbf{R}} e^{s 4^{\frac{1}{s}} h^{-\frac{1}{s}}|x|^{\frac{1}{s}}-\frac{1}{2} x^{2}} \leqslant C_{h, s} h^{k} k !^{s}, \quad k \in \mathbf{N},
$$

for a new constant $C_{h, s}>0$.

The corrected result concerns operators with Schwartz kernel of the oscillatory integral form

$$
K_{T}(x, y)=(2 \pi)^{-(d+N) / 2} \sqrt{\operatorname{det}\left(\begin{array}{cc}
p_{\theta \theta}^{\prime \prime} / i & p_{\theta y}^{\prime \prime} \\
p_{x \theta}^{\prime \prime} & i p_{x y}^{\prime \prime}
\end{array}\right)} \int_{\mathbf{R}^{N}} e^{i p(x, y, \theta)} d \theta \in \mathscr{S}^{\prime}\left(\mathbf{R}^{2 d}\right)
$$

where $x, y \in \mathbf{R}^{d}$. Here $p$ is a quadratic form on $\mathbf{R}^{2 d+N}$ associated with the positive Lagrangian that is defined by the twisted graph of the matrix $T \in \operatorname{Sp}(d, \mathbf{C})$ which is assumed to be positive in the sense of [3, Eq. 5.10]. The kernel is, modulo sign, independent of the form $p$ and the dimension $N$, thanks to the the factor in front of the integral [3, p. 444].

Proposition 8.1 Suppose $T \in \mathrm{Sp}(d, \mathbf{C})$ is positive and let $\mathscr{K}_{T}: \mathscr{S}\left(\mathbf{R}^{d}\right) \rightarrow \mathscr{S}^{\prime}\left(\mathbf{R}^{d}\right)$ be the continuous linear operator having Schwartz kernel $K_{T} \in \mathscr{S}^{\prime}\left(\mathbf{R}^{2 d}\right)$ defined by (8.1). For $s>1 / 2$ the operator $\mathscr{K}_{T}$ is continuous on $\Sigma_{s}\left(\mathbf{R}^{d}\right)$ and $\mathscr{K}_{T}$ extends uniquely to a continuous operator on $\Sigma_{s}^{\prime}\left(\mathbf{R}^{d}\right)$.

Proof By [3, Proposition 5.10] (cf. [2]) the matrix $T$ can be factorized as

$$
T=\chi_{1} T_{0} \chi_{2}
$$

where $\chi_{1}, \chi_{2} \in \operatorname{Sp}(d, \mathbf{R}), T_{0} \in \operatorname{Sp}(d, \mathbf{C})$ is positive and $(y, \eta)=T_{0}(x, \xi), x, \xi, y, \eta \in$ $\mathbf{R}^{d}$, where for each $1 \leqslant j \leqslant d$ we have either

$$
\left(\begin{array}{l}
y_{j} \\
\eta_{j}
\end{array}\right)=\left(\begin{array}{cc}
\cosh \tau_{j} & -i \sinh \tau_{j} \\
i \sinh \tau_{j} & \cosh \tau_{j}
\end{array}\right)\left(\begin{array}{l}
x_{j} \\
\xi_{j}
\end{array}\right)
$$

with $\tau_{j} \geqslant 0$, or

$$
\left(\begin{array}{l}
y_{j} \\
\eta_{j}
\end{array}\right)=\left(\begin{array}{ll}
1 & 0 \\
i & 1
\end{array}\right)\left(\begin{array}{l}
x_{j} \\
\xi_{j}
\end{array}\right)
$$

By [3, Proposition 5.9] we have

$$
\mathscr{K}_{T}= \pm \mu\left(\chi_{1}\right) \mathscr{K}_{T_{0}} \mu\left(\chi_{2}\right) .
$$


According to Proposition $4.4, \mu\left(\chi_{j}\right)$ is continuous on $\Sigma_{s}\left(\mathbf{R}^{d}\right)$, so it remains to show that $\mathscr{K}_{T_{0}}$ is continuous on $\Sigma_{s}\left(\mathbf{R}^{d}\right)$.

The matrix $T_{0}$ can be factorized as

$$
T_{0}=T_{1} T_{2} \cdots T_{d}
$$

where the matrices $T_{j}, 1 \leqslant j \leqslant d$, commute pairwise, and have the following structure. It holds $(y, \eta)=T_{j}(x, \xi)$ where $\left(y_{k}, \eta_{k}\right)=\left(x_{k}, \xi_{k}\right), k \in\{1,2, \cdots, d\} \backslash\{j\}$ and either (3) for some $\tau_{j} \geqslant 0$, or (4) holds.

Again by [3, Proposition 5.9]

$$
\mathscr{K}_{T_{0}}= \pm \mathscr{K}_{T_{1}} \mathscr{K}_{T_{2}} \cdots \mathscr{K}_{T_{d}}
$$

and thus it suffices to show that $\mathscr{K}_{T_{j}}$ of each of the stated two types is continuous on $\Sigma_{s}\left(\mathbf{R}^{d}\right)$. In order to do that we first identify the operators $\mathscr{K}_{T_{j}}$, cf. [2, p. 297].

Suppose $(y, \eta)=T_{j}(x, \xi)$ where $\left(y_{k}, \eta_{k}\right)=\left(x_{k}, \xi_{k}\right), k \in\{1,2, \cdots, d\} \backslash\{j\}$. Case (i) Suppose (3) for some $\tau_{j} \geqslant 0$. Define the symmetric block matrix

$$
Q_{j}=\frac{1}{2}\left(\begin{array}{cc}
e_{j} e_{j}^{t} & 0 \\
0 & e_{j} e_{j}^{t}
\end{array}\right) \in \mathbf{R}^{2 d \times 2 d}
$$

where $e_{j} \in \mathbf{R}^{d}$ denotes the standard basis vector with zero entries except for position $j$ which is one. With $F_{j}=\mathcal{J} Q_{j}$ a short calculation shows that

$$
e^{-2 i \tau_{j} F_{j}}=T_{j}
$$

which reveals that $\mathscr{K}_{T_{j}}$ is the solution operator to the initial value Cauchy problem (5.1) when the Hamiltonian Weyl symbol is defined by

$$
q_{j}(x, \xi)=\left\langle(x, \xi), Q_{j}(x, \xi)\right\rangle=\frac{1}{2}\left(x_{j}^{2}+\xi_{j}^{2}\right), \quad(x, \xi) \in T^{*} \mathbf{R}^{d}
$$

at time $\tau_{j}$, that is $\mathscr{K}_{T_{j}}=e^{-\tau_{j} q_{j}^{w}(x, D)}$. Here $q_{j}^{w}(x, D)=\frac{1}{2}\left(x_{j}^{2}+D_{j}^{2}\right)$ is the Hermite operator (harmonic oscillator) acting on variable $j$, divided by two.

For this operator the Hermite functions are eigenfunctions, and

$$
\frac{1}{2}\left(x_{j}^{2}+D_{j}^{2}\right) h_{\alpha}=\left(\alpha_{j}+\frac{1}{2}\right) h_{\alpha}, \quad \alpha \in \mathbf{N}^{d}
$$

(cf. e.g. [5]). By the uniqueness of the solution to the Cauchy problem (5.1) we have

$$
\mathscr{K}_{T_{j}} h_{\alpha}=e^{-\frac{\tau_{j}}{2}\left(x_{j}^{2}+D_{j}^{2}\right)} h_{\alpha}=e^{-\tau_{j}\left(\alpha_{j}+\frac{1}{2}\right)} h_{\alpha}, \quad \alpha \in \mathbf{N}^{d} .
$$


Using the seminorms on $\Sigma_{s}\left(\mathbf{R}^{d}\right)$ defined by Hilbert sequence spaces $\ell_{s, r}^{2}\left(\mathbf{N}^{d}\right)$, cf. (1), and the orthonormality of $\left\{h_{\beta}\right\}_{\beta \in \mathbf{N}^{d}} \subseteq L^{2}\left(\mathbf{R}^{d}\right)$ we obtain for $f \in \Sigma_{s}\left(\mathbf{R}^{d}\right)$ and $\alpha \in \mathbf{N}^{d}$

$$
\left(\mathscr{K}_{T_{j}} f, h_{\alpha}\right)=\sum_{\beta \in \mathbf{N}^{d}}\left(f, h_{\beta}\right)\left(\mathscr{K}_{T_{j}} h_{\beta}, h_{\alpha}\right)=\left(f, h_{\alpha}\right) e^{-\tau_{j}\left(\alpha_{j}+\frac{1}{2}\right)},
$$

and hence for any $r>0$

$$
\begin{aligned}
\left\|\left(\mathscr{K}_{T_{j}} f, h_{\alpha}\right)_{\alpha \in \mathbf{N}^{d}}\right\|_{\ell_{s, r}^{2}}^{2} & =\sum_{\alpha \in \mathbf{N}^{d}}\left|\left(\mathscr{K}_{T_{j}} f, h_{\alpha}\right)\right|^{2} e^{2 r|\alpha|^{\frac{1}{2 s}}} \\
& =\sum_{\alpha \in \mathbf{N}^{d}}\left|\left(f, h_{\alpha}\right)\right|^{2} e^{-\tau_{j}\left(2 \alpha_{j}+1\right)} e^{2 r|\alpha|^{\frac{1}{2 s}}} \\
& \leqslant \sum_{\alpha \in \mathbf{N}^{d}}\left|\left(f, h_{\alpha}\right)\right|^{2} e^{2 r|\alpha|^{\frac{1}{2 s}}} \\
& =\|\left(f, h_{\alpha}\right)_{\alpha \in \mathbf{N}^{d} \|_{\ell_{s, r}^{2}}^{2}}
\end{aligned}
$$

This shows the continuity $\mathscr{K}_{T_{j}}: \Sigma_{s}\left(\mathbf{R}^{d}\right) \rightarrow \Sigma_{s}\left(\mathbf{R}^{d}\right)$.

Case (ii) Suppose (4). Define the symmetric block matrix

$$
Q_{j}=\frac{1}{2}\left(\begin{array}{cc}
e_{j} e_{j}^{t} & 0 \\
0 & 0
\end{array}\right) \in \mathbf{R}^{2 d \times 2 d}
$$

and $F_{j}=\mathcal{J} Q_{j}$. Then

$$
e^{-2 i F_{j}}=T_{j}
$$

which implies that $\mathscr{K}_{T_{j}}$ is the solution operator to the initial value Cauchy problem (5.1) when the Hamiltonian Weyl symbol is defined by

$$
q_{j}(x, \xi)=\left\langle(x, \xi), Q_{j}(x, \xi)\right\rangle=\frac{1}{2} x_{j}^{2}
$$

at time $t=1$, that is $\mathscr{K}_{T_{j}}=e^{-q_{j}^{w}(x, D)}$. Since $q_{j}^{w}(x, D) f(x)=\frac{x_{j}^{2}}{2} f(x)$ we have $\mathscr{K}_{T_{j}} f(x)=e^{-\frac{1}{2} x_{j}^{2}} f(x)$ which is a Gaussian multiplicator operator with respect to variable $j$.

From the Lemma we obtain for any $\alpha, \beta \in \mathbf{N}^{d}$ and any $h>0$ using the seminorms (2.3)

$$
\begin{aligned}
\left|x^{\beta} D^{\alpha}\left(e^{-\frac{1}{2} x_{j}^{2}} f(x)\right)\right| & \leqslant \sum_{\gamma_{j} \leqslant \alpha_{j}}\left(\begin{array}{c}
\alpha_{j} \\
\gamma_{j}
\end{array}\right)\left|D^{\gamma_{j}}\left(e^{-\frac{1}{2} x_{j}^{2}}\right)\right|\left|x^{\beta} D^{\alpha-\gamma_{j} e_{j}} f(x)\right| \\
& \lesssim\|f\|_{\mathcal{S}_{s, h}} \sum_{\gamma_{j} \leqslant \alpha_{j}}\left(\begin{array}{l}
\alpha_{j} \\
\gamma_{j}
\end{array}\right) h^{\gamma_{j}} \gamma_{j} !^{!}\left(\beta !\left(\alpha-\gamma_{j} e_{j}\right) !\right)^{s} h^{|\beta|+|\alpha|-\gamma_{j}}
\end{aligned}
$$




$$
\begin{aligned}
& \leqslant\|f\|_{\mathcal{S}_{s, h}} h^{|\alpha+\beta|}(\beta ! \alpha !)^{s} 2^{\alpha_{j}} \\
& \leqslant\|f\|_{\mathcal{S}_{s, h}}(2 h)^{|\alpha+\beta|}(\beta ! \alpha !)^{s}, \quad x \in \mathbf{R}^{d},
\end{aligned}
$$

and thus

$$
\left\|e^{-\frac{1}{2} x_{j}^{2}} f\right\|_{\mathcal{S}_{s, h}} \lesssim\|f\|_{\mathcal{S}_{s, h / 2}}
$$

We have shown the continuity of $\mathscr{K}_{T_{j}}: \Sigma_{s}\left(\mathbf{R}^{d}\right) \rightarrow \Sigma_{s}\left(\mathbf{R}^{d}\right)$.

\section{References}

1. Carypis, E., Wahlberg, P.: Propagation of exponential phase space singularities for Schrödinger equations with quadratic Hamiltonians. J. Fourier Anal. Appl. 23, 530-571 (2017)

2. Hörmander, L.: $L^{2}$ estimates for Fourier integral operators with complex phase. Ark. Mat. 21(2), 283307 (1983)

3. Hörmander, L.: Symplectic classification of quadratic forms, and general Mehler formulas. Math. Z. 219(3), 413-449 (1995)

4. Langenbruch, M.: Hermite functions and weighted spaces of generalized functions. Manuscr. Math. 119, 269-285 (2006)

5. Wong, M.W.: The heat equation for the Hermite operator on the Heisenberg group. Hokkaido Math. J. 34(2), 393-404 (2005)

Publisher's Note Springer Nature remains neutral with regard to jurisdictional claims in published maps and institutional affiliations. 